\title{
Density functional theory with nonlocal correlation: A key to the solution of the CO adsorption puzzle
}

\author{
P. Lazić, ${ }^{1,2}$ M. Alaei ${ }^{1,3}$ N. Atodiresei, ${ }^{1,4}$ V. Caciuc,,${ }^{5,1}$ R. Brako, ${ }^{2}$ and S. Blügel ${ }^{1}$ \\ ${ }^{1}$ Institut für Festkörperforschung (IFF) and Institute for Advanced Simulation (IAS), Forschungszentrum Jülich, 52425 Jülich, Germany \\ ${ }^{2}$ Rudjer Bošković Institute, Zagreb, Croatia \\ ${ }^{3}$ Department of Physics, Isfahan University of Technology, Isfahan 84156, Iran \\ ${ }^{4}$ The Institute of Scientific and Industrial Research, Osaka University, 567-0047 Osaka, Japan \\ ${ }^{5}$ Physikalisches Institut, Westfälische Wilhelms Universität Münster, Wilhelm-Klemm-Str. 10, 48149 Münster, Germany
}

(Received 13 October 2008; revised manuscript received 9 September 2009; published 5 January 2010)

\begin{abstract}
We study the chemisorption of CO molecule into sites of different coordination on (111) surfaces of late $4 d$ and $5 d$ transition metals. In an attempt to solve the well-known CO adsorption puzzle, i.e., discrepancies of adsorption site preferences with experiment which appear in the standard density functional theory calculations, we have applied the relatively new van der Waals-density functional of nonlocal correlation. In all considered cases this reduces or completely solves the site preference discrepancies and improves the value of the adsorption energy. By introducing a cutoff distance for nonlocal interaction we can pinpoint the length scale at which the correlation plays a major role in the systems considered.
\end{abstract}

DOI: 10.1103/PhysRevB.81.045401

PACS number(s): 68.43.Bc, 71.15.Mb

\section{INTRODUCTION}

For decades the density functional theory (DFT) (Ref. 1) has been the main theoretical tool used to analyze, understand, and predict material properties and chemical processes. Although very successful in explaining the finest details of many systems, even very complex ones, it fails in some cases, in particular, in weakly bound systems where van der Waals forces play a major role. Examples are numerous. Practically all biological systems and any system containing noble gas atoms cannot be calculated correctly within the current approximations to the DFT, the local-density approximation (LDA), and the semilocal generalized gradient approximation (GGA). One solution is to resort to methods beyond the DFT approach, either phenomenological or quantum-chemistry post-Hartree-Fock $a b$ initio methods, the most accessible being the perturbational MP2. ${ }^{2,3}$ An alternative approach was recently proposed by Dion et al., ${ }^{4,5}$ who developed a new exchange-correlation functional named van der Waals-density functional (vdW-DF), which is parameter free and therefore is considered ab initio in true spirit of DFT as it depends only upon the total electron density $n(\boldsymbol{r})$. The main idea of the vdW-DF is the introduction of nonlocal correlation into DFT calculations.

The failure of local and semilocal functionals in weakly bounded systems is expected but what is puzzling is that current approximations to the DFT fail to predict the correct adsorption site of CO molecules on several (111) metal surfaces, of which the Pt is the most famous example. Adsorption of $\mathrm{CO}$ on Pt surface stands as an archetype for catalytic reactions and is of tremendous practical importance, in particular, in exhaust catalyst in the car industry. Some eight years ago Feibelman et al. ${ }^{6}$ published a comprehensive study of $\mathrm{CO}$ on $\mathrm{Pt}(111)$, performing calculations independently with several state-of-the-art DFT computer codes, for a variety of exchange-correlation potentials. They found that DFT systematically predicts that $\mathrm{CO}$ prefers the hollow site on $\mathrm{Pt}(111)$ surface while experiments show the adsorption into the top, sometimes bridge but never hollow site. Due to expected success of DFT in such chemisorbed systems the problem was named the CO adsorption puzzle. The same puzzle appears on some other (111) surfaces such as $\mathrm{Cu}$ and $\mathrm{Rh}$. Even more confusing is the correct prediction of the hollow site on some other surfaces such as Pd(111) in agreement with experiment. Additionally to the site preference, getting the correct barrier height between the sites of different coordination is of enormous importance for the analysis of diffusion phenomena on surfaces as discussed by $\mathrm{Hu}$ et $a l^{2}$ By considering the puzzle problem they have also noticed that the lack of nonlocal correlation is one of the possible sources of the problem.

The most recent DFT studies of the puzzle problem, using other GGA functionals such as BLYP (Ref. 7) and hybrid functionals such as B3LYP and HSE (Ref. 8) are inconclusive on whether this kind of approach can give systematic improvements. On the other hand, an analysis based on comparison with quantum-chemistry calculations of $\mathrm{CO}$ adsorbed on finite clusters of atoms leads to the conclusion that the errors in present-day DFT exchange-correlation functionals are of short range. ${ }^{2}$

There has been some discussion (see, e.g., Ref. 3) on the relative merits of the DFT and wave-function approaches, and how well the DFT can describe the puzzle systems at all. In principle, the answer to the last question is clear. Since the $\mathrm{CO}$ adsorption site in puzzle systems is a ground-state property, the Hohenberg-Kohn theorems ensure that DFT can find the correct answer, provided a good enough density functional is used. This does not mean that any of the present-day functionals, be it LDA, GGA, hybrid, or empirically corrected, is appropriate, and the problem should be approached with an open mind. Improving the functional but in the true spirit of the density functional approach allowing it to depend upon the total electron density $n(\boldsymbol{r})$ only (although not necessarily just through local and gradient terms), can prove to be of enormous practical importance. Due to computational complexity, quantum-chemistry wave-function-based 
approaches can hardly scale to system sizes necessary for this kind of problems. On the other hand, one should keep in mind that properties such as the highest occupied molecular orbital-lowest unoccupied molecular orbital gap, often discussed in the context of quantum-chemistry approaches which aim to describe correctly the empty and excited electronic states, are of very limited relevance in DFT, where the Kohn-Sham wave functions are auxiliary quantities without any guarantee of the accuracy of empty states.

\section{II. vdW-DF CORRELATION FUNCTIONAL}

Up to now vdW-DF was mostly used to treat van der Waals type of systems, achieving quite good success. ${ }^{9,10}$ However, the functional itself was constructed in a seamless fashion, i.e., describing the nonlocal correlation correctly at all length scales. In fact, the functional has recently been successfully applied to a covalently bonded system. ${ }^{11}$ Here we use the functional in order to treat the puzzle systems. We apply the vdW-DF as a postprocessing procedure on a charge density obtained from a standard GGA calculation. Such an application of the functional has been justified by the work of Thonhauser et al., ${ }^{12}$ who implemented the functional in a fully self-consistent manner into a DFT code and showed that the rearrangement of the total electron density was negligible.

Following the original procedure of Dion et $a l .{ }^{4}$ we replace the conventional GGA correlation energy by a sum of two terms, the LDA correlation and a newly defined nonlocal term $E_{\mathrm{c}}^{\mathrm{nl}}$,

$$
E_{\mathrm{vdW}-\mathrm{DF}}=E_{\mathrm{GGA}}-E_{\mathrm{GGA}, \mathrm{c}}+E_{\mathrm{LDA}, \mathrm{c}}+E_{\mathrm{c}}^{\mathrm{nl}},
$$

where $E_{\mathrm{GGA}}$ is the self-consistent GGA total energy. The last term in Eq. (1) is completely determined by the electronic charge density and requires the evaluation of a double space integral,

$$
E_{\mathrm{c}}^{\mathrm{nl}}=\frac{1}{2} \int d^{3} r d^{3} r^{\prime} n(\boldsymbol{r}) \phi\left(\boldsymbol{r}, \boldsymbol{r}^{\prime}\right) n\left(\boldsymbol{r}^{\prime}\right),
$$

where $n(\boldsymbol{r})$ is the charge density at point $\boldsymbol{r}$ and $\phi\left(\boldsymbol{r}, \boldsymbol{r}^{\prime}\right)$ is a kernel function described in Ref. 4. Since to our knowledge there is no available code to calculate the vdW-DF we have developed our own. ${ }^{13}$ The code has been tested on a few examples which have been published so far, ${ }^{4}$ achieving a complete agreement.

It has been argued by the authors of the vdW-DF and others ${ }^{4,11}$ that the vdW-DF should be used together with the revPBE functional for the calculation of the exchange energy contribution because it seems to approximate most correctly the exact exchange and by that avoids some spurious binding effects. We have used both PBE (Ref. 14) and revPBE (Ref. 15) exchange functionals in our DFT calculations. In Sec. V we return to the question of the most appropriate exchange functional.

\section{III. vdW-DF CALCULATIONS OF CO ON METAL SURFACES}

We have applied the vdW-DF to the adsorption of CO into top and fcc hollow sites on a range of (111) surfaces which have been related to the $\mathrm{CO}$ puzzle, for two different coverages. Our $a b$ initio DFT calculations were carried out mostly by employing the pseudopotential plane-wave formulation as implemented in the PWSCF code. ${ }^{16}$ In the spirit of the original puzzle article of Feibelman et al. ${ }^{6}$ we also performed calculations for the experimentally most interesting coverage of 1/4 CO monolayer employing a second electronic structure code, the VASP code, ${ }^{17-19}$ a code which implements the projector-augmented wave method. ${ }^{20}$ The plane-wave energy cutoff was $40 \mathrm{Ry}$, i.e., $544 \mathrm{eV}$ in PWSCF calculations, and from $500 \mathrm{eV}$ up to $1000 \mathrm{eV}$ for checking purposes in VASP. We used three layers of metal atoms and a $k$-point mesh of $4 \times 4 \times 1$ in PWSCF calculations at $1 / 12$ coverage, four layers and $8 \times 8 \times 1 k$ points at $1 / 4$, the top two atomic layers being allowed to relax. In VASP calculations five layers (three relaxing) and a $k$-point mesh of $4 \times 4 \times 1$ were used. $\mathrm{CO}$ molecules were adsorbed on one side, with $13 \AA$ or more vacuum above. The double sum of the nonlocal correlation [the discretised version of Eq. (2)] was evaluated within the unit cell and with $\boldsymbol{r}^{\prime}$ in the eight neighboring cells, i.e., the parameters perl, per2, and per3 in JUNOLO (Ref. 13) were 1, 1 , and 0 . We verified that these values were sufficient by running test calculations with larger values of the parameters.

The results are summarized in Table I. The most important results are listed in column six. We immediately notice that the application of vdW-DF lowers the energy of adsorption, $E_{\text {ads }}$, into the top site of the (111) transition-metal surface, $E_{\text {ads }}^{\text {top }}$, by roughly $100 \mathrm{meV}$ as compared to the one at the hollow site, $E_{\text {ads }}^{\text {hollow }}$. In the case of $\mathrm{Rh}$ this completely resolves the puzzle, i.e., $E_{\text {ads }}^{\text {top }}<E_{\text {ads }}^{\text {hollow }}$. The adsorption of $\mathrm{CO}$ into the hollow site on $\mathrm{Pd}$ is the only case in agreement with experiment prior to the application of vdW-DF, and the hollow site correctly remains more stable after the application of vdW-DF. For Pt we find that the shift of energy depends on the coverage $\theta$. While for $\theta=1 / 12$ the shift toward the on-top position is about $100 \mathrm{meV}$, for a coverage of $\theta=1 / 4$ the shift is about $45 \mathrm{meV}$. For the coverage of $\theta=1 / 4$ the shift is slightly too small to resolve the puzzle and a small preference for the hollow site still remains even if the revPBE exchange is used, confirming the reputation of $\mathrm{CO} / \mathrm{Pt}(111)$ as the most difficult puzzle system. Furthermore, in all cases the adsorption energy is improved compared to the pure GGA value.

An important message is that this energy shift is basically independent of the first-principles method or DFT functional used. In general, this is different for the energy difference, $E_{\text {ads }}^{\text {top }}-E_{\text {ads }}^{\text {hollow }}$, between on-top and hollow-site adsorptions of $\mathrm{CO}$ or the absolute values of the adsorption energies $E_{\text {ads }}$. These energy differences depend on the method or functional. However, our results are in good agreement with previous PWSCF results ${ }^{7}$ and recent VASP results. ${ }^{22}$

In order to understand the difference between the PBE (or any GGA) and the vdW-DF correlation functional we have examined the spatial distribution of the correlation energy densities. For each of the two functionals we have calculated the correlation energy density for the CO chemisorbed on metal, and subtracted the values for the clean surface and an isolated molecule at exactly the same positions as in the coupled system, in order to analyze in which regions of 
TABLE I. Energies of CO adsorption, $E_{\text {ads }}$, on (111) surfaces of several metals, calculated with the standard GGA and the vdW-DF postprocessing approaches. All energies are in meV. In columns 4 and 5, the differences of the adsorption energies into the top, $E_{\text {ads }}^{\text {top }}$, and the threefold fcc hollow, $E_{\text {ads }}^{\text {hollow }}$, sites are given, calculated with the standard GGA functional (the specific functional is specified in column 3) and the vdW-DF postprocessing approaches, respectively. The difference of the two results in column 6 shows that the application of vdW-DF always shifts the balance toward the top site. The two rows marked with the asterisk in the "Functional" column have been calculated by evaluating the revPBE exchange on densities obtained using the PBE exchange functional. The entries shown in bold give adsorption site preference in agreement with the experimental assignment shown in the last column. The columns 7-9 give the calculated adsorption energies and the experimental values, where available.

\begin{tabular}{|c|c|c|c|c|c|c|c|c|}
\hline \multirow[b]{2}{*}{ Surface } & \multicolumn{2}{|c|}{ DFT GGA } & \multicolumn{2}{|c|}{$E_{\text {ads }}^{\text {top }}-E_{\text {ads }}^{\text {hollow }}$} & \multirow[b]{2}{*}{ Shift } & \multicolumn{3}{|c|}{$E_{\text {ads }}^{\text {top }}$} \\
\hline & Code & Functional & GGA & vdW-DF & & GGA & vdW-DF & Experiment (Ref. 21) \\
\hline \multicolumn{9}{|c|}{ Coverage $\theta=1 / 12$} \\
\hline $\mathrm{Pt}$ & PWSCF & PBE & 198 & 95 & -103 & -1648 & -1442 & Top \\
\hline $\mathrm{Pt}$ & PWSCF & revPBE & 93 & -3 & -96 & -1331 & -1135 & \\
\hline $\mathrm{Rh}$ & PWSCF & PBE & 49 & -101 & -150 & & & \\
\hline $\mathrm{Pd}$ & PWSCF & PBE & 631 & 526 & -105 & & & fcc hollow \\
\hline \multicolumn{9}{|c|}{ Coverage $\theta=1 / 4$} \\
\hline $\mathrm{Pt}$ & PWSCF & PBE & 170 & 120 & -50 & -1630 & -1460 & $-1370 \pm 130$ top \\
\hline $\mathrm{Pt}$ & PWSCF & revPBE & 60 & 20 & -40 & -1326 & -1160 & \\
\hline $\mathrm{Pt}$ & VASP & PBE & 100 & 56 & -44 & -1572 & -1373 & \\
\hline $\mathrm{Pt}$ & VASP & revPBE* & 45 & 1 & -44 & -1323 & -1124 & \\
\hline $\mathrm{Cu}$ & PWSCF & PBE & 150 & 50 & -100 & -800 & -720 & $-500 \pm 50$ top \\
\hline $\mathrm{Cu}$ & PWSCF & revPBE & 20 & -30 & -50 & -540 & -520 & \\
\hline $\mathrm{Cu}$ & VASP & PBE & 139 & 34 & -105 & -679 & -620 & \\
\hline $\mathrm{Cu}$ & VASP & revPBE* & 73 & -32 & -105 & -425 & -366 & \\
\hline $\mathrm{Rh}$ & PWSCF & PBE & 30 & -100 & -130 & -1890 & -1840 & $-1450 \pm 140$ top \\
\hline
\end{tabular}

space changes occur upon chemisorption. Since the PBE correlation is semilocal, i.e., depends upon the charge density and the gradient of it while the vdW-DF correlation is truly nonlocal by virtue of the double space integral (2), their spatial distribution is quite different. In Fig. 1 we show the difference of the two correlation energy densities. In the red regions the PBE correlation overbinds compared to vdW-DF while in blue regions it underbinds. Integration over space shows that the total effect is always an overbinding by $\mathrm{PBE}$ but it is more pronounced in the fcc hollow case than in the top case, by about $100 \mathrm{meV}$, as discussed in Table I. The figure suggests that this is due to the fact that there are three regions of overbinding in the fcc hollow case and only one in the top case (although the latter is somewhat stronger than any single hollow one).

We conclude that the PBE correlation functional makes a quantitative error in evaluating correlation energies of different adsorption sites in these systems and therefore seems to yield wrong results, e.g., due to the failure to correctly predict adsorption sites in seemingly chemically bonded systems - this was the puzzle. PBE otherwise correctly captures the physics of the problem as our relaxation tests for puzzle systems of $\mathrm{Pt}$ and $\mathrm{Cu}$ show. We have relaxed the $\mathrm{CO}$ molecule by straightforward total-energy minimization rather than by force calculations. We first changed the position of the molecule relative to the surface in the perpendicular direction while keeping the $\mathrm{C}-\mathrm{O}$ distance at the relaxed value

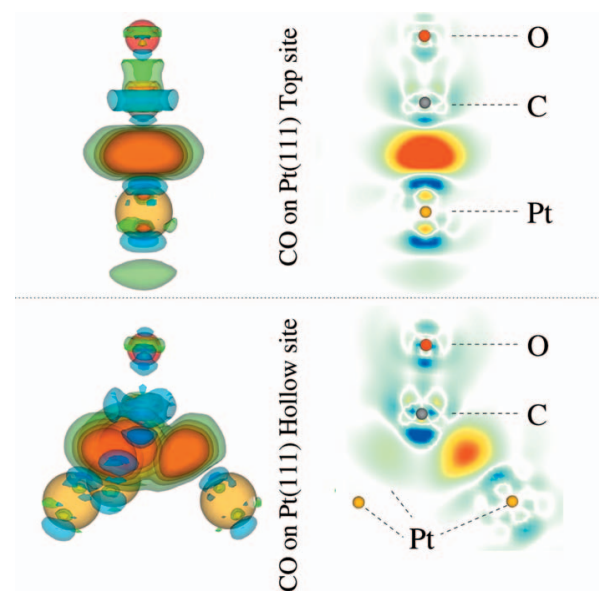

FIG. 1. (Color) Differences in correlation energy densities between $\operatorname{PBE}\left(E_{\mathrm{PBE}, \mathrm{c}}\right)$ and vdW-DF $\left(E_{\mathrm{LDA}, \mathrm{c}}+E_{\mathrm{c}}^{\mathrm{nl}}\right)$ calculations, for top site, upper panel, and hollow site, lower panel. The threedimensional representation (left) and the cut along the CO molecule and through the nearest metal atom of the Pt surface (right) are shown. Red (blue) colors show regions in which PBE yields larger (smaller) binding energies compared to the vdW-DF. Color scales are the same in both images, with green representing zero value. By summing all the contributions we found out that PBE correlation overbinds compared to vdW-DF in both cases. This is shown in more details in the supplementary material. ${ }^{23}$ 


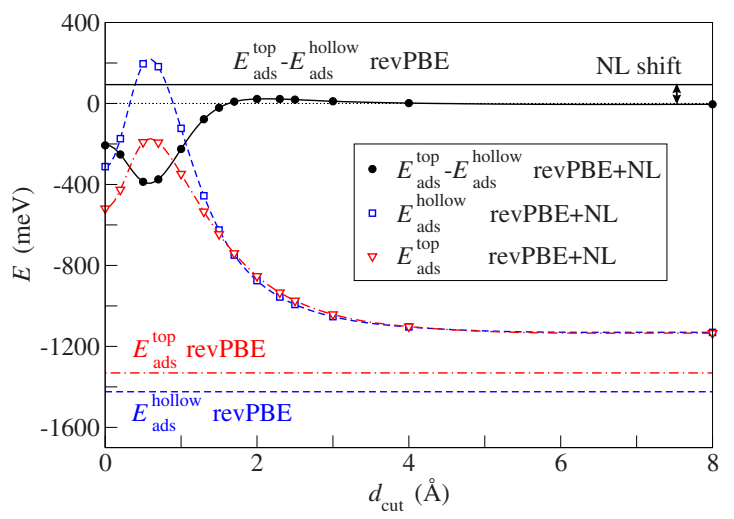

FIG. 2. (Color online) The energies of adsorption of a CO molecule at top and hollow sites on the Pt(111) surface at a coverage of $1 / 12$, and the difference of the two. The calculation was done using the density obtained in a revPBE GGA calculation, varying the cutoff in Eq. (3). At $d_{\text {cut }}=0$ (i.e., including GGA revPBE exchange but only the LDA term in correlation) the top site is clearly preferred. The full revPBE $+\mathrm{NL}$ (large $d_{\text {cut }}$ ) gives a small preference for the top site while the plain GGA shows a strong preference for the hollow site, at variance with experiment.

of the GGA calculation, and later we also changed that distance. It turns out that the position of $\mathrm{CO}$ determined in GGA calculations is the optimal one also for the vdW-DF. Moreover, the curvature of the adsorption energy around the minimum does not change if we apply vdW-DF although the value of the energy changes. In weakly bound systems in which the PBE does not describe the physics correctly, due to the lack of the van der Waals interaction, as, for example, a $\mathrm{Kr}$ dimer, both parameters - the position of energy minimum and the curvature around it-are strongly changed upon the application of the vdW-DF. ${ }^{24}$

\section{NONLOCAL CORRELATION WITH CUTOFF}

In order to further investigate how the nearsightedness of electron correlation translates into the distance dependence of the nonlocality in the vdW-DF correlation we have performed calculations of $E_{\mathrm{c}}^{\mathrm{nl}}\left(d_{\text {cut }}\right)$ in which we have introduced a cutoff distance $d_{\text {cut }}$ in Eq. (2) by weighting the spatial distances by a step function,

$$
\theta\left(d_{\text {cut }}-\left|\boldsymbol{r}-\boldsymbol{r}^{\prime}\right|\right),
$$

where the cutoff $d_{\text {cut }}$ defines the largest distance at which the nonlocal correlation is included.

We performed calculations for $\mathrm{CO}$ chemisorbed on $\mathrm{Pt}(111)$ in top and hollow sites at equilibrium distance, and subtracted the energy for the separated molecule and surface, all calculated with the same value of $d_{\text {cut }}$. The results are shown in Fig. 2. The adsorption energies at $d_{\text {cut }}=0$ correspond to the rather unphysical approximation of taking both the local (LDA) and semilocal (revPBE) terms for the exchange but only the local term for the correlation. The energy of adsorption into the top site is lower but the ordering is reversed when the full semilocal PBE correlation term is added, the straight lines near the bottom of the graph, in disagreement with the experiment. If instead the nonlocal vdW-DF correlation is gradually added, three regimes are clearly visible. At small $d_{\text {cut }}$, less than $\sim 1 \AA$, the contribution to the nonlocal correlation comes from within regions where charge is accumulated or depleted upon chemisorption (roughly corresponding to regions of intense colors in Fig. $1)$. This region is still somewhat unphysical because in the integral (2) only a small part of the kernel $\phi$ contributes. In the region $\sim 2-3 \AA$, which is the characteristic length of the chemical bond (and of the distances between blue blobs in Fig. 1) the nonlocal correlation becomes more binding. These changes occur faster for the hollow than for the top site, reflecting the fact that there are three bonding regions instead of one. Finally, in the true van der Waals region, say for $d_{\text {cut }}>4 \AA$, the interaction is, of course, attractive but this region contributes little to the strongly chemisorbed system of $\mathrm{CO}$ on $\mathrm{Pt}(111)$, and almost nothing to the difference between the top and hollow sites.

Figure 2 shows that the physical length scale of the nonlocal correlation energy, i.e., when the energies and the energy differences saturate, is on the order of the distance between the carbon and the substrate atoms. However, at this length scale the exact geometry of the chemisorbed complex becomes relevant, which intuitively explains why the GGA, which depends only upon the local value of the density gradient and thus does not see the big picture, is not able to give the correct values, in particular, the energy differences between sites of different coordination.

\section{DISCUSSION}

In the whole paper we made little reference to the exchange energy but followed the arguments of the authors of the vdW-DF, and considered also the revPBE exchange functional, which indeed shifts the preference toward adsorption into top sites. In a recent article Gulans et al. ${ }^{25}$ have reexamined the use of exchange functionals in conjunction with vdW-DF correlation, and concluded that revPBE is usually more advantageous but that in some cases, in particular, for hydrogen-bonded complexes, there is systematic underbinding when using the revPBE while the PBE exchange functional succeeds well.

In a paper examining the adsorption of $\mathrm{CO}$ on $\mathrm{Cu}, \mathrm{Rh}$, and $\mathrm{Pt}(111)$ surfaces in top and hollow sites using hybrid exchange functionals with the conventional PBE correlation, Stroppa et $a l{ }^{26}$ found that the hollow site is destabilized by 50-150 meV. Again, this is not sufficient to give the correct site preference in the case of $\mathrm{CO}$ on $\mathrm{Pt}(111)$. The downside of the hybrid exchange functionals is that they further increase the already overestimated adsorption energies. (The fact that the PBE functional as well as other GGA functionals overbind the adsorption of molecules on metal surfaces was realized some time ago. ${ }^{27}$ ) Our calculations show that the vdW-DF correlation acts in the direction of destabilizing the hollow site and at the same time decreasing the adsorption energies. It is thus plausible that combining the vdW-DF correlation and the exact exchange or any hybrid exchange functionals can bring all calculated properties of the puzzle systems in agreement with experiment. In any case, the ques- 
tion of the most appropriate exchange functional beyond $\mathrm{PBE}$ or revPBE to be used in conjunction with vdW-DF correlation deserves further study.

In this paper we may have applied the seamless nonlocal vdW-DFT to a system which may not have been at the focus of applicability when the functional had been made originally. Of course, nobody really knows the range of applicability and the seamless nature of the functional seems to make the range rather wide. Support of the potential power of nonlocal functional for correlation comes from two recently published papers. ${ }^{22,28}$ The exact exchange and random-phase approximation (RPA) correlations have been used in a postprocessing scheme starting from DFT calculations, rather similar to our approach. The method was applied to the adsorption of $\mathrm{CO}$ on $\mathrm{Cu}(111)$, as well as to calculate properties of several other strongly bound systems. The preference of $\mathrm{CO}$ for the on-top adsorption and an improvement of the adsorption energy were obtained. In all cases considered, the exact exchange seems to combine well with the nonlocal correlation. The RPA correlation is also nonlocal in nature but carries a large computational cost and Harl et $a .^{22}$ concluded to move into the direction of nonlocal-density functionals that rely on a description of the correlation energy in the RPA. In this publication we anticipated the application of such a nonlocal correlation function to this kind of problems.

In summary, we have found that for the strongly bound systems considered here, the geometry of the equilibrium configuration and the curvature of the adsorption potential, i.e., the bond distances and the vibrational frequencies, are not changed noticeably upon the application of the nonlocal vdW-DF. The adsorption energies, however, change by amounts larger than the desired "chemical accuracy" of 1 $\mathrm{kcal} / \mathrm{mol}$ (i.e., $43.4 \mathrm{meV}$ ). Even more importantly, the change is different for competing $\mathrm{CO}$ adsorption sites of different coordination. We have shown that in this respect the $\mathrm{vdW}-\mathrm{DF}$ always acts in the right direction, and resolves a large part of the $\mathrm{CO}$ adsorption puzzle, i.e., in almost all cases we achieve a preference of the top site, although larger energy differences would be required to agree with experimental thermal stability. We have shown that nonlocal correlation effects at a typical length scale of carbon-metal-atom distance $(\sim 2-3 \AA)$ are decisive for a good description of $\mathrm{CO}$ puzzle systems.

\section{CONCLUSIONS}

To conclude, our calculations show that the vdW-DF theory improves the electron correlation in systems which were initially not expected to be much affected by it. In particular, it reduces significantly the error in the bindingenergy differences between competing atomic configurations with different coordination. Looking beyond the adsorption of $\mathrm{CO}$ on $\mathrm{Pt}(111)$ and similar surfaces, a systematic improvement of the accuracy of the DFT calculations in this direction would be crucial for the proper description of microscopic processes of growth, materials transport, and chemical reactivity and thus may impact into many fields of the sciences and engineering. Surprisingly the impact of the nonlocal functional in the DFT is much larger than just to enable treatment of vdW systems. It seems that it is a general step forward compared to currently most popular local/semilocal functionals and it comes at a reasonable computational cost.

\section{ACKNOWLEDGMENTS}

The calculations were performed on the JUMP and Blue/ Gene supercomputers at the Forschungszentrum Jülich, Germany. Two of us, P.L. and S.B., thank the Deutsche Forschungsgemeinschaft (DFG) (Priority Programme "Molecular Magnetism") and Alexander von Humboldt foundation for financial support. N.A. acknowledges the support of Japan Society for the Promotion of Science. R.B. acknowledges the support of MSES of the Republic of Croatia through Project No. 098-0352828-2836EPS.
${ }^{1}$ P. Hohenberg and W. Kohn, Phys. Rev. 136, B864 (1964).

${ }^{2}$ Q. M. Hu, K. Reuter, and M. Scheffler, Phys. Rev. Lett. 98, 176103 (2007); 99 169903(E) (2007).

${ }^{3}$ S. Sharifzadeh, P. Huang, and E. Carter, J. Phys. Chem. C 112, 4649 (2008).

${ }^{4}$ M. Dion, H. Rydberg, E. Schröder, D. C. Langreth, and B. I. Lundqvist, Phys. Rev. Lett. 92, 246401 (2004); 95, 109902(E) (2005).

${ }^{5}$ D. C. Langreth, M. Dion, H. Rydberg, E. Schröder, P. Hyldgaard, and B. I. Lundqvist, Int. J. Quantum Chem. 101, 599 (2005).

${ }^{6}$ P. J. Feibelman, B. Hammer, J. K. Nørskov, F. Wagner, M. Scheffler, R. Stumpf, R. Watwe, and J. Dumesic, J. Phys. Chem. B 105, 4018 (2001).

${ }^{7}$ M. Alaei, H. Akbarzadeh, H. Gholizadeh, and S. de Gironcoli, Phys. Rev. B 77, 085414 (2008).

${ }^{8}$ A. Stroppa and G. Kresse, New J. Phys. 10, 063020 (2008).

${ }^{9}$ S. D. Chakarova-Käck, E. Schröder, B. I. Lundqvist, and D. C.
Langreth, Phys. Rev. Lett. 96, 146107 (2006).

${ }^{10}$ D. C. Langreth, B. I. Lundqvist, S. D. Chakarova-Käck, V. R. Cooper, M. Dion, P. Hyldgaard, A. Kelkkanen, J. Kleis, Lingzhu Kong, Shen Li, P. G. Moses, E. Murray, A. Puzder, H. Rydberg, E. Schröder, and T. Thonhauser, J. Phys.: Condens. Matter 21, 084203 (2009).

${ }^{11}$ K. Johnston, J. Kleis, B. I. Lundqvist, and R. M. Nieminen, Phys. Rev. B 77, 121404(R) (2008).

${ }^{12}$ T. Thonhauser, V. R. Cooper, S. Li, A. Puzder, P. Hyldgaard, and D. C. Langreth, Phys. Rev. B 76, 125112 (2007).

${ }^{13}$ Jülich Non Local—JUNOLO code, http://www.fz-juelich.de/iff/ src/th1/JuNoLo

${ }^{14}$ J. P. Perdew, K. Burke, and M. Ernzerhof, Phys. Rev. Lett. 77, 3865 (1996).

${ }^{15}$ Y. Zhang and W. Yang, Phys. Rev. Lett. 80, 890 (1998).

${ }^{16} \mathrm{http} / / /$ www.pwscf.org/

${ }^{17}$ G. Kresse and J. Hafner, Phys. Rev. B 47, 558 (1993). 
${ }^{18}$ G. Kresse and J. Furthmüller, Phys. Rev. B 54, 11169 (1996).

${ }^{19} \mathrm{http} / / / \mathrm{cms} . \mathrm{mpi}$.univie.ac.at/vasp/

${ }^{20}$ P. E. Blöchl, Phys. Rev. B 50, 17953 (1994).

${ }^{21}$ F. Abild-Pedersen and M. P. Andersson, Surf. Sci. 601, 1747 (2007).

${ }^{22}$ J. Harl and G. Kresse, Phys. Rev. Lett. 103, 056401 (2009).

${ }^{23}$ See supplementary material at http://link.aps.org/supplemental/ 10.1103/PhysRevB.81.045401 for video that explains construction of cross sections in Fig. 1 which enables 3D view of the structure of the FCC case.
${ }^{24}$ P. Lazić, R. Brako, and B. Gumhalter, J. Phys.: Condens. Matter 19, 305004 (2007).

${ }^{25}$ A. Gulans, M. J. Puska, and R. M. Nieminen, Phys. Rev. B 79, 201105(R) (2009).

${ }^{26}$ A. Stroppa, K. Termentzidis, J. Paier, G. Kresse, and J. Hafner, Phys. Rev. B 76, 195440 (2007).

${ }^{27}$ B. Hammer, L. B. Hansen, and J. K. Nørskov, Phys. Rev. B 59, 7413 (1999).

${ }^{28}$ X. Ren, P. Rinke, and M. Scheffler, Phys. Rev. B 80, 045402 (2009). 